\title{
THE PROFILE OF SOCIAL MEDIA USERS IN ROMANIA: INDIVIDUAL CHARACTERISTICS AND THE NUMBER OF SOCIAL CONNECTIONS
}

\author{
Alexandru Razvan FLOREA \\ Bucharest University of Economic Studies, Romania \\ alflorea@ea.com \\ Monica ROMAN \\ Bucharest University of Economic Studies, Romania \\ monica.roman@csie.ase.ro
}

\begin{abstract}
The information revolution has dramatically changed the way of human living. In this study, we explain how a powerful open source tool, like $R$, can be leveraged to collect and examine data from social networks. Using advanced machine learning algorithms on social media data, we provided a first snapshot of the Romanian Twitter users' demographics, and we also validated recent social theories on the dimension of social networks, such as Dunbar's number. Among many techniques, we used API calls to connect $R$ with Twitter, face recognition algorithms provided by Azure, and traditional statistical tests like Chi-Squared. Our results show that Romanian Twitter users are young, mostly males, and reside in the largest share in the capital of the country, Bucharest. Moreover, the research tests and confirms the Dunbar's number, showing that physical appearance, like smiling in Twitter profile pictures, is positively associated with the number of the social connections, for both males and females.
\end{abstract}

Keywords: social behavior, data collection, computer programs, social media, R, face recognition

JEL classification: J11, C8, L86

DOI: $10.12948 / \mathrm{ie} 2019.04 .21$

\section{Introduction}

Over the past four decades, computers have reshaped the way we communicate, learn, meet each other, and many other elements of our day-to-day life. The technology redefined individuals' lifestyle so dramatically that life without computers becomes harder to imagine with every day.

The growth of technology impacted all fields, including social activities. More and more people use virtual environments for social interactions such as communication, learning, meeting new people, getting the news, etc. The interest in using datasets from the online platforms to study social activities and trends increases every year. There are numerous researches done on the US social behavior, such as human mobility, happiness, cultural understanding or health status [1] [2] [3]. However, there are less studies focused on understanding the population of social media users and their characteristics in less developed countries, such as Romania.

Back in the 1992, Robin Dunbar predicted that human beings cannot maintain more than 150 stable relationships [4] The theory was validated by numerous researches ref, and scientists took the theory to the next level, showing that laughter is highly correlated with the social bond of an individual. 
Our overall aim on this paper is to start understanding the particularities of the Romanian Twitter users and their behavior. Moreover, we want to validate Dunbar's theory regarding social bonds and laughter by testing the correlation between the smile in the profile picture and the number of twitter Friends. Our methodology on answering these questions will include using various techniques, from descriptive statistics to advanced image processing algorithms. We structure our paper in 5 main sections. In the first two sections, we briefly present how social media platforms were leveraged in research and we also explain the data and techniques that are going to be used to answer our questions.

In the third section, we examine the details of the population of Romanian Twitter users and in the fourth section we apply conventional statistical techniques on non-conventional datasets to validate and confirm social behaviors and trends.

In the last part, we provide a summary of the conclusions and discuss possible next steps for future researches.

\section{Social network platforms: a reliable data provider}

Social Networks platforms passed the volatile periods, and the possibility became a certainty in less than two decades. The maturity of the industry is so high that the top five most popular social platforms did not recently change. On the other hand, the frequency and purposes of internet usage vary in different parts of the world ${ }^{1}$.

With billions of real-time social media interactions, the analytical potential of these platforms is undoubtful high [5] [6]. Twitter is an online social platform on which users post messages known as "tweets"2. It is also available to anyone and there is no doubt that it is one of the most successful communication platforms. However, despite the enormous amount of information that Twitter can provide, these datasets are not representative for the whole population, but they can be used as proxies for regional social patterns such as human mobility [7] [8].

Twitter data unlocked major opportunities as it can be used to serve various purposes. Some papers offered ways to predict the demographics of social media users using information related to whom they follow [1].In the same time, studying demographic details, such as age, gender or ethnic characteristics might provide insights about how and why certain groups tweet. In other words, some researchers use Twitter data to understand better the marginalized groups or even the cultures of different ethnic groups [3].

Other inquiries combined the Twitter data with other data sources in a very creative and effective way and obtained astonishing results, such us happiness, education or even obesity levels at the regional level [2]. Some researchers took the same approach and combined traditional Census data with Twitter data to drive more insights and explain better users' online behavior.

For example, it has been found that "cognitive factors and offline characteristics affect the quality of online interactions", meaning that "places where social media users engage more deeply with less diverse social contacts, are those where they express more negative emotions, like sadness and anger and also have residents with lower household income and education levels" while "places where people engage less frequently but with diverse contacts have

\footnotetext{
${ }^{1}$ Dave Chaffey: Global social media research summary; 2019; link: https://www.smartinsights.com/social-media-marketing/social-media-strategy/new-global-social-mediaresearch/

${ }^{2}$ Definition of Twitter and tweets: https://help.twitter.com/en/new-user-faq
} 
happier, more positive messages posted from them and also have better educated, younger, more affluent residents"3 [9]

The Twitter data is now used even in the Health Sector and more and more researches receive funds to develop new ways to use these datasets [10] [11]. In the same time, more and more fields, such as election predictions, marketing, human mobility or demand planning, start to use Twitter data [12] [13].

The online social network is arguably the largest observational instrument of human behavior nowadays. Moreover, Twitter data is tremendously big, and it is easily accessible by social scientists. However, we understand that there are technical and legal limitations ${ }^{4}$ regarding the use of this data [14]. Therefore, we will consider them in our analysis, and no data will be stored at the user level.

\section{Methods and techniques}

Our analysis will rely on R, RStudio, and Microsoft Office to perform the data collection, statistical tests, and visualization elements. $\mathrm{R}$ is a programming language and an exceptional environment for analytical computing or graphics producing ${ }^{5}$.

The connection between our local $\mathrm{R}$ environment and Twitter was established by using the official $\mathrm{API}^{6}$ provided by Twitter. An API is a collection of protocols and routines that facilitate the communication among multiple software engines.

Face Recognition is the result of the emergence of multiple disciplines, such as computer vision, pattern identification, image processing or neural networks. Face Recognition Technology (FRT) has vast applicability as it can be used in multiple fields. It is already used in the process of matching real-time images with passports photos. Other studies leveraged FRT to estimate demographic details based on the available photos provided by various databases [15].

All the computer disciplines develop at an incredible pace. In 2015, the face recognition systems achieved a new accuracy record of $99.65 \%$ on Labeled Faces in the Wild (LFW) data set [16]. This paper will leverage the incredible expertise and processing power of Azure in the face recognition field ${ }^{7}$.

In the process of trying to understand better how the Romanian Twitter Population looks like from a demographic perspective, we will mainly use descriptive statistics at a regional NUTS $\mathrm{I}^{8}$ level. The correlations between social trends and theories and social media data will be tested by using the Chi Squared test.

\section{Understanding the demographics of Romanian residents who use Twitter}

The algorithm proposed in the previous section returned $\sim 280.000$ geo-located tweets, all of them being posted from somewhere near a city in Romania. Based on these tweets, we identified $\sim 60.000$ unique users. There are big chances that most of these 60.000 users to be Romanian residents, but to increase the accuracy of our results, we've filtered the residents of

\footnotetext{
3 "Emotions, Demographics and Sociability in Online Interactions", 2018, Conference: INTERNATIONAL AAAI CONFERENCE ON WEBLOGS AND SOCIAL MEDIA

${ }^{4}$ The EU General Data Protection Regulation (GDPR) is the most important change in data privacy regulation in 20 years: https://eugdpr.org/

${ }^{5} \mathrm{R}$ is a free software environment for statistical computing and graphics. It compiles and runs on a wide variety of UNIX platforms, Windows and MacOS (https://www.r-project.org/)

${ }^{6}$ Application programming interface

${ }^{7}$ The Azure Face API is a cognitive service that provides algorithms for detecting, recognizing, and analyzing human faces in images.

${ }^{8}$ Nomenclature of Territorial Units for Statistics
} 
www.conferenceie.ase.ro

Romania using the self-declared permanent location. After cleaning the data, we've obtained around 8.000 unique users.

The next step was to estimate the user's demographic characteristics by analyzing their profile pictures. We used the advanced and powerful tool provided by Azure and obtained relevant results for more than 3.500 unique users.

This paper will focus on analyzing these 3.500 unique users as they seem to represent a reliable population in terms of engagement, residency, and demographic characteristics. On the other hand, it is essential to understand that these 3.500 users do not represent the entire population of Romanian Twitter users.

We first examine the age of the Romanian twitter users. Figure 1 shows that the distribution of users by age is positively skewed with a mean of 33 and a median of 32 . At the same time, the below histogram let us understand that most of the collected Twitter users are between 16 and 50 years old.

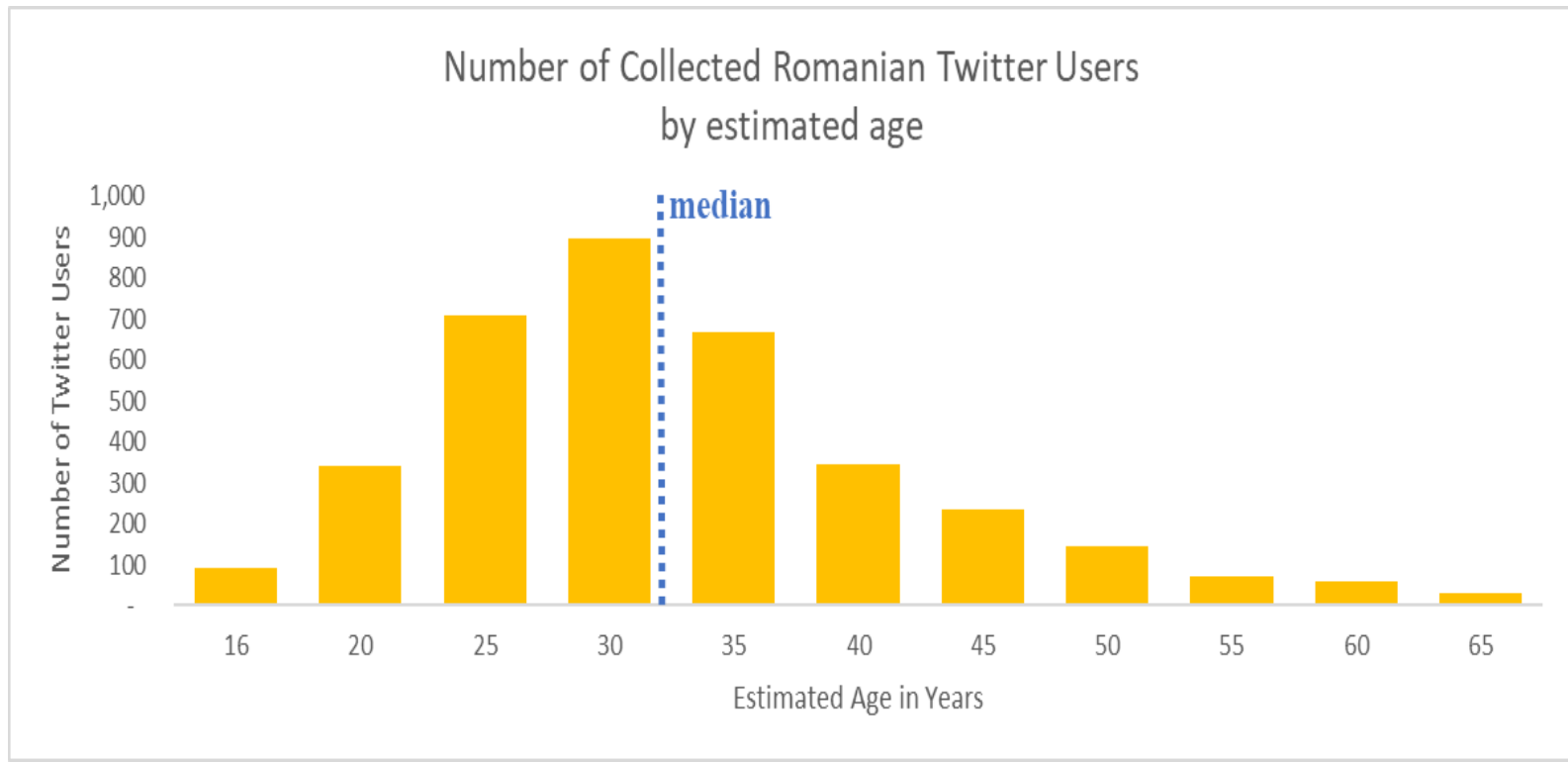

Figure 1. Twitter users by estimated age

Source: Graph produced by authors

While more than $65 \%$ of the collected users seem to be men, the women users, with an average age of 30 , tend to be slightly younger than the population of men users that have an average age of 35. The first purpose of our research is to better understand the Romanian population of Twitter users and the differences between them across regions. Therefore, we looked at the average age across regions and concluded that there are not major differences across regions in terms of the age of the users as the average age of the users is between 30 and 35 in all NUTS I regions.

Another important aspect that contributes to the better understanding of the Romanian Social networks is the popularity of Twitter across the NUTS I regions of Romania. As expected, the biggest concentration of Twitter users is around the capital of the country (Bucharest-Ilfov region). While the North-Vest is the second region in terms of its popularity, Twitter seems to be less utilized in the South-East and South-Muntenia regions.

Nevertheless, the Figure 2 Indicates that Bucharest-Ilfov and West are the regions with the most engaged Twitter users, counting more than $45 \%$ of our total population. However, it is important to understand that even if there are regional differences in terms of popularity and 
engagements, Twitter is used as an effective social media platform across all Romanian regions.

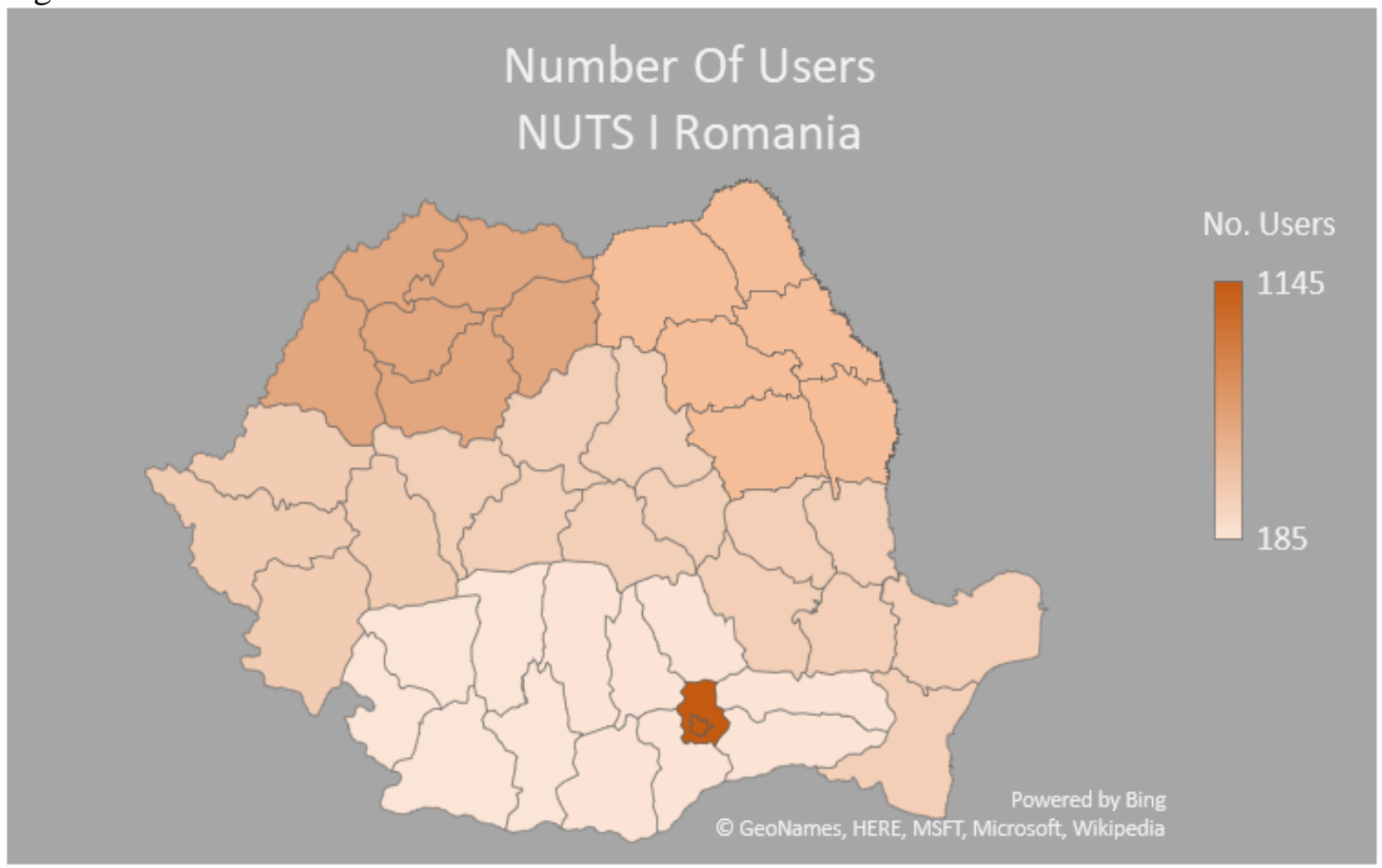

Figure 2. Twitter users by NUTS I Regions

Source: Graph produced by authors

Some studies suggest that "laughter, through an endorphin-mediated opiate effect, may play a crucial role in social bonding"9 [17].

\section{Is smile a driver for social bonds?}

Two decades ago, Robin Dunbar, a popular British anthropologist, noticed that the size of the primate brain size is directly correlated with their social groups. In other words, bigger brains lead to larger social groups. This striking discovery led Dunbar to an extraordinary prediction: a human being can have a maximum of 150 stable relationships [4]. Since the 1990s, a lot of studies ${ }^{10}$ were conducted to validate this theory and it has turned out that social groups and networks, including the online ones, follow Dunbar's number [18] [19]. Moreover, the famous anthropologist, Robin Dunbar, continued his work and showed that laughing might be a significant driver for the social bonding [17].

This paper aims to validate this theory by investigating how strongly the smile of users correlates with their social activity. Dunbar suggests that a person can have a maximum 150 stable relationships. At the same time, during one of his presentations ${ }^{11}$ he indicated that the limit should be adjusted to 120-130 for the online social platforms as not of the people own an online account. Therefore, since a Twitter user can entertain a maximum of 130 online

\footnotetext{
${ }^{9}$ Proceedings Biol Sci. 2012- Social laughter is correlated with an elevated pain threshold (https://www.ncbi.nlm.nih.gov/pubmed/21920973)

${ }^{10}$ MIT Technology Review: https://www.technologyreview.com/s/601369/your-brain-limits-you-to-just-fivebffs/

${ }^{11}$ TEDxObserver - Robin Dunbar - Can the internet buy you more friends?, 2012, https://www.youtube.com/watch?v=071pED729k8
} 
relationships, we flagged everyone with more than 100 friends as a person with high Social Activity.

The results presented in Figure 3 indicate that there is a significant correlation between women who smile in the profile picture and their Social Activity. In other words, women who tend to smile in the profile picture also tend to have more social bonds.

Chi Squared Test:

Null hypothesis: there is no association between the two variables.

Alternative hypothesis: there is an association between the two variables. $p$-value $<0.05=>$ the null hypothesis can be rejected for a confidence interval of $95 \%$

$$
\text { Pearson's Chi-squared test with Yates' continuity correction }
$$

data: smile_pop

$\mathrm{X}$-squared $=4.4608, \mathrm{df}=1, \mathrm{p}$-value $=0.03468$

Figure 3. Female population, Smile vs High Social Activity Source: Output produced by authors using $R$

Using the same logic as above, we ran the test for the male population and got similar results. Men who tend to smile in the profile picture also tend to have more twitter friends. $p$-value $<0.05=>$ the null hypothesis can be rejected for a confidence interval of $95 \%$

\section{Pearson's Chi-squared test with Yates' continuity correction}

data: smile_pop

$\mathrm{X}$-squared $=5.237, \mathrm{df}=1, \mathrm{p}$-value $=0.02211$

Figure 4. Male population, Smile vs High Social Activity

Source: Output produced by authors using $R$

\section{Conclusion}

The increase in size and accuracy of social media data unlocks more and more research opportunities and facilitates robust and innovative approaches to study recent social behaviors. Although collecting and examining data from social media platforms represent a significant challenge, we believe these alternative data sources might notably contribute to the study of social activity. Our paper provided a close view of the demographic characteristics of Romanian Twitter users.

Moreover, our research proved that facial expressions or appearances are essential for social bonding not only in real life but also in the virtual environment.

In other words, it validated that smiling or laughing is correlated with the number of social bonds.

This inquiry on Romanian social behavior can serve as a foundation for more detailed studies on demographics and social trends, like migration.

This paper showed that social media data could provide valuable information which could be leveraged to understand the latest social patterns.

We aim to continue our research and explore the online behavior of Twitter users in other meaningful directions.

The next direction we plan to address includes the exploration of the behavioral variations among Twitter users with different levels of education. 
www.conferenceie.ase.ro

\section{References}

[1] A. Culotta, N. K. Ravi and J. Cutler, "Predicting the Demographics of Twitter Users from Website Traffic Data," in Proceedings of the Twenty-Ninth AAAI Conference on Artificial Intelligence, Austin, Texas, USA, 2015.

[2] L. Mitchell, M. R. Frank, K. D. Harris, P. S. Dodds and C. M. Danforth, "The Geography of Happiness: Connecting Twitter Sentiment and Expression, Demographics, and Objective Characteristics of Place," Plos One , vol. 8, no. 5, 2013.

[3] D. Murthy, A. Gross and A. Pensavalle, "Urban Social Media Demographics: An Exploration of Twitter Use in Major American Cities," Journal of Computer-Mediated Communication, vol. 21, no. 1, pp. 33-49, 2016.

[4] R. I. M. Dunbar, "Neocortex size as a constraint on group size in primates," Journal of Human Evolution, vol. 22, no. 6, 1992.

[5] MacEachren, Robinson, Jaiswal, Pezanowski, Savelyev, Blanford and Mitra, "Geo-Twitter Analytics: Applications in Crisis Management," Proceedings, 25th International Cartographic Conference, Paris, France, 2011.

[6] A. Florea and M. Roman, "An ocean of migration flows data has been revealed-using social media for migration research," in IE 2018-The 17th International Conference on Informatics in Economy, Iasi, Romania, 2018.

[7] B. Hawelka, I. Sitko, E. Beinat, S. Sobolevsky, P. Kazakopoulos and C. Ratti, "Geo-located Twitter as proxy for global mobility pattern," Global Networks- International Journal of Geographical Information Science Vol. 00, No. 00, 2014.

[8] E. Zagheni, V. R. K. Garimella, I. Weber and B. State, "Inferring International and Internal Migration Patterns from Twitter data," Stanford University, 2014.

[9] K. Lerman, M. Arora, L. Gallegos, P. Kumaraguru and D. Garcia, "Emotions, Demographics and Sociability in Twitter," in International AAAI Conference on Weblogs and Social Media, Palo Alto, California, USA, 2018.

[10] L. Sinnenberg, A. Buttenheim, K. Padrez, C. Mancheno, L. Ungar and R. Merchant, "Twitter as a Tool for Health Research: A Systematic Review," American Journal of Public Health (AJPH), vol. 107, no. 1, 2017.

[11] L. Sinnenberg, C. L. DiSilvestro and C. Mancheno, "Twitter as a Potential Data Source for Cardiovascular Disease Research," JAMA Cardiol, vol. 1, no. 9, pp. 1032-1036, 2016.

[12] E. Sanders, M. d. Gier and A. v. d. Bosch, "Using Demographics in Predicting Election Results with Twitter," in International Conference on Social Informatics, Seattle, Washington, USA, 2016.

[13] F. Luo, G. Cao, K. Mulligan and X. Lib, "Explore spatiotemporal and demographic characteristics of human mobility via Twitter: A case study of Chicago," Applied Geography, vol. 70, pp. 11-25, 2017.

[14] T. H. McCormick, H. Lee, N. Cesare, A. Shojaie and E. S. Spiro, "Using Twitter for Demographic and Social Science Research: Tools for Data Collection and Processing," Sociological Methods \& Research, vol. 46, no. 3, pp. 390-421, 2017.

[15] A. Florea and M. Roman, "Using Face Recognition with Twitter Data for the Study of International," Informatica Economică, vol. 22, no. 4, pp. 31-46, 2018.

[16] F. Schroff, D. Kalenichenko and J. Philbin, "FaceNet: A Unified Embedding for Face Recognition and Clustering," in IEEE Xplore, 2015.

[17] R. Dunbar, R. Baron, A. Frangou, E. Pearce, E. van Leeuwen, J. Stow, G. Partridge, I. MacDonald, V. Barra and M. van Vugt, "Social laughter is correlated with an elevated pain threshold," in Proceedings Biological Sciences, 2012.

[18] P. MacCarron, K. Kaski and R. Dunbar, "Calling Dunbar's Numbers," Social Networks, vol. 47, pp. 151-155, 2016. 
[19] B. Gonçalves, N. Perra and A. Vespignani, "Modeling Users' Activity on Twitter Networks: Validation of Dunbar's Number," Plos One, vol. 6, no. 8, 2011. 